\title{
Momentos-chave e natureza das intervenções do terapeuta em psicoterapia breve psicodinâmica
}

\author{
Eduardo Khater - Pontificia Universidade Católica de Campinas, São Paulo, Brasil \\ Evandro Morais Peixoto - Pontifícia Universidade Católica de Campinas, São Paulo, Brasil \\ Giovanna Corte Honda - Pontifícia Universidade Católica de Campinas, São Paulo, Brasil \\ Maria Leonor Espinosa Enéas - Universidade Presbiteriana Mackenżie, São Paulo, Brasil \\ Elisa Medici Pĩão Yoshida - Pontifícia Universidade Católica de Campinas, São Paulo, Brasil
}

\begin{abstract}
Resumo
Objetivou-se avaliar possível associação entre a natureza das intervenções do psicoterapeuta e as respostas da paciente em momentos-chave de três fases do processo. Metodologicamente, trata-se de pesquisa de processo de caso único, de caráter exploratório. Analisaram-se transcrições das sessões do início ( $1^{\mathrm{a}}$ e $2^{\mathrm{a}}$ sessões), meio ( $6^{\mathrm{a}}$ e $7^{\mathrm{a}}$ sessões) e fim $\left(10^{\mathrm{a}}\right.$ e $11^{\mathrm{a}}$ sessões de psicoterapia breve psicodinâmica, com o Modelo dos Ciclos Terapêuticos (TCM) e classificação das Intervenções Terapêuticas (IT). O TCM é um método computadorizado de análise que identifica momentos-chave do processo, denominados ciclos terapêuticos. As IT foram classificadas em expressivas e suportivas. Os resultados sugeriram que as intervenções são moduladas pelas respostas do paciente e pelas tarefas específicas do terapeuta, independentemente da fase do processo. Também não se observou associação entre os ciclos terapêuticos e a modalidade de intervenção. Algumas limitações, concernentes aos instrumentos de avaliação utilizados, são apontadas como possíveis fatores de viés.

Palavras-chaves: Mudança (psicologia); Software; Aplicações do computador; Processos psicoterapêuticos.
\end{abstract}

Key moments and nature of therapeutic interventions in brief psychodynamic psychotherapy

\begin{abstract}
The study aimed to evaluate possible associations between nature of the therapist intervention and the patient response, in key-moments of different phases of the psychotherapeutic process. This is a case-based research, with an exploratory character. We have analyzed transcripts of early sessions (1st and 2nd sessions), middle (6th and 7th sessions) and end (10th and 11th sessions) of brief psychodynamic psychotherapy, with Model of Cycles Therapeutic (TCM) and classification of Therapeutic Interventions (IT). TCM is a computerized method of analysis that identifies key moments of the process, named therapeutic cycles. The IT were classified into expressive and supportive. The results suggest that interventions are modulated by the responses of the patient and the specific tasks of the therapist, regardless of stage. Also no association was observed between cycles and therapeutic modality of intervention. Some limitations concerning the applied instruments are pointed as source of possible bias. Keywords: Psychotherapy change (psychology); Software; Computer applications; Psychotherapeutic processes.
\end{abstract}

\section{Momentos-clave y naturaleza de las intervenciones del terapeuta en psicoterapia psicodinámica breve}

\section{Resumen}

El objetivo del estudio fue evaluar la posible asociación entre la naturaleza de las estrategias de intervención del psicoterapeuta y las respuestas del paciente en momentos clave de tres etapas del proceso. Metodológicamente, es una investigación de estudio de caso único, de carácter exploratorio. Se analizaron las transcripciones de las primeras sesiones $\left(1^{\mathrm{a}}\right.$ y $\left.2^{\mathrm{a}}\right)$, medio $\left(6^{\mathrm{a}}\right.$ y $\left.7^{\mathrm{a}}\right)$ y al final $\left(10^{\mathrm{a}}\right.$ y $\left.11^{\mathrm{a}}\right)$ de psicoterapia psicodinámica breve con el Modelo de Ciclos Terapéuticos (TCM) y clasificación de las intervenciones terapéuticas (IT). El TCM es un método computarizado de análisis que identifica los momentos clave del proceso, denominados ciclos terapéuticos. Las IT se clasificaron en expresivas y de apoyo. Los resultados sugieren que las intervenciones son hechas en función de las respuestas del paciente y de las tareas específicas del terapeuta, independientemente de la etapa del proceso. Además, no se observó asociación entre los ciclos y modalidades de la intervención terapéutica. Algunos límites con relación a los instrumentos de evaluación utilizados, son apuntados como posibles factores de sesgo.

Palabras-clave: Cambio (psicología); Software; Aplicaciones de la computadora; Procesos psicoterapéuticos.

\section{Introdução}

As pesquisas realizadas nas últimas décadas têm apontado que os resultados de psicoterapias dependem de inúmeros fatores, que podem ser didaticamente divididos em variáveis do paciente, variáveis do terapeuta e da relação entre eles (Angus e colaboradores, 2010; Castonguay \& Beutler, 2005). Para fins de pesquisas empíricas, essas variáveis são usualmente isoladas e estudadas de forma independente ou, em alguns casos, de forma combinada (Botella e colaboradores, 2008). No presente artigo, variáveis do terapeuta (modalidade de intervenção) foram cotejadas com as respostas do paciente, em momentos considerados clinicamente significantes (momento-chave) de três fases do processo: inicial, medial e final.

Em psicoterapias breves psicodinâmicas, tem especial relevância a capacidade do terapeuta de 
responder de forma apropriada a cada momento vivido pelo paciente (Enéas \& Yoshida, 2012). Isso quer dizer que ele deve ajustar "o tom" de suas intervenções ao momento específico do paciente (Mergenthaler, 2008). Efetivamente, a experiência clínica e de pesquisa mostram que um processo de psicoterapia não é homogêneo (Krause e colaboradores, 2007) e pode ser dividido, de forma algo arbitrária, em fase inicial, medial e final, sendo que cada uma delas é caracterizada por aspectos que têm sido descritos, grosso modo, da seguinte forma: fase inicial, caracterizada pela exposição da queixa e/ou motivo da busca pelo atendimento de aspectos da vida do paciente de forma a fornecer um quadro geral de seu desenvolvimento emocional e principais dificuldades. Nesse primeiro momento, o terapeuta tende a mostrar-se interessado pelos problemas do paciente, suas vivências e experiências de vida, num esforço de compreendê-lo e de construir as bases para o estabelecimento de uma boa aliança terapêutica e definição do foco (Fernandez e colaboradores, 2008; Messer \& Warren, 1995).

$\mathrm{Na}$ fase medial da psicoterapia, espera-se que paciente e terapeuta já estejam tratando especificamente do conflito, ou conflitos, que levaram o paciente a buscar o auxílio profissional. O intuito é o de compreender os fatores que estariam impedindo o paciente a superar suas dificuldades, tanto as de ordem consciente quanto inconsciente, levando-o à elaboração do conflito focal (Enéas \& Yoshida, 2012). E, na fase final, a principal tarefa é a de consolidar as mudanças e progressos obtidos e trabalhar o desligamento. Isto é, escrutinar os sentimentos suscitados pelo término do atendimento e ajudar o paciente a enfrentá-los com o menor sofrimento possível. Ademais, é importante ajudá-lo a se imaginar em situações futuras, quando não contará mais com o apoio do terapeuta para responder a situações semelhantes às que o levaram a buscar a terapia. Desse modo, as especificidades de cada fase vão demandar do terapeuta diferentes modalidades de intervenção (Enéas \& Rocha, 2011).

De acordo com Luborsky (1984), as intervenções na prática das psicoterapias psicodinâmicas podem ser classificadas sob um continuum suportivo-expressivo. As intervenções de natureza "suportiva" objetivam a manutenção do nível de funcionamento do paciente. São técnicas de apoio, que demonstram o quanto o psicoterapeuta compreende as situações do paciente. As intervenções de natureza "expressivas" têm como objetivo facilitar a comunicação e compreensão, por parte do paciente, de seus problemas e conflitos inconscientes.
A divisão das intervenções em suportivas e expressivas foi resultado de projeto de pesquisa da Fundação Menninger (Menninger Clinic Treatment Interventions Project), dispostas segundo um continum reproduzido por Gabbard (1994/1998). Com base nesse continuum e também na classificação sugerida por Fiorini (1995), Yoshida, Gatti, Enéas, e Coelho Filho (1997) propuseram um sistema classificatório de intervenções de acordo com o qual aquelas de natureza expressiva incluem: interpretação, confrontação, clarificação e assinalamento; as de natureza suportiva abrangem: validação empática, conselho e elogio, fornecer informações e recapitulação. E, além disso, há também outras, de natureza neutra, que compreendem: encorajamento a elaborar, afirmação, interrogação, meta-intervenção e dar enquadramento. Este continum foi adotado na presente pesquisa e as definições de cada categoria de intervenção são apresentadas no item instrumentos do método.

Voltando para os fatores envolvidos em um processo terapêutico, cabe ainda destacar que as evidências de mudança do paciente não aparecem de forma linear e regular ao longo de todo o processo, mas se apresentam associadas a momentos específicos, tidos como clinicamente significantes. Dentre as propostas de conceituação e de identificação desses momentos, destaca-se aqui o Modelo dos Ciclos Terapêuticos (Therapeutic Cycles Model - TCM) (Mergenthaler, 1996; 2008). O TCM é um método de análise de textos por computador que permite identificar momentos chave na narrativa do paciente, do terapeuta, ou de ambos em interação. O termo "momento-chave, refere-se a uma ou mais sessões do tratamento ou ao segmento de uma sessão, vistos como clinicamente importantes" (Mergenthaler, 1996, p. 1306). O TCM está baseado no entendimento de que a expressão de emoções é um fator central de todo processo terapêutico. Todavia, a ela deve estar associada a capacidade de perceber e refletir sobre a experiência vivida e de expressá-la verbalmente. Ou seja, esse modelo parte do pressuposto que o processo de mudança terapêutica é conduzido pela interação entre experiência emocional e cognitiva do paciente e do terapeuta.

A versão do TCM adaptada para o português (Yoshida \& Mergenthaler, 2011) conta, como na versão original (Mergenthaler, 1996), com um programa de análise de dados por computador (Cycles Model - CM), que mede a ocorrência de palavras das transcrições de sessões psicoterapêuticas. Isso é feito a partir do confronto com um dicionário pré-desenvolvido para a língua portuguesa, com palavras categorizadas segundo 
a natureza do tom emocional (positiva ou negativa), ou que indiquem se tratar de uma abstração (positiva, negativa ou neutra). A leitura do texto, pelo software, fornece a frequência de palavras associadas a cada uma das categorias de emoção ou de abstração, o que permite uma análise qualitativa que se traduz em padrões de emoção-abstração (Mergenthaler, 1996).

Para as análises, o texto é usualmente dividido em blocos de 150 palavras, que são avaliados quanto ao tom emocional e uso de abstrações, e gráficos representativos do padrão predominante em cada bloco, são gerados. Os gráficos permitem que seja realizada uma análise para a determinação de padrões clinicamente relevantes durante processos de psicoterapias (gráficos ilustrativos podem ser encontrados, por exemplo, em Yoshida \& Mergenthaler, 2011). Quando se identificam os padrões utilizados pelo paciente, pelo terapeuta ou ambos em interação, é possível observar a ocorrência de ciclos, que Mergenthaler (1996) define como ciclos terapêuticos. Ou seja, uma "sequência temporal específica dos quatro padrões de emoção-abstração (p. 1308)" (relaxamento, reflexão, experiência e conexão), cada um correspondendo a uma fase do ciclo terapêutico.

O TCM já foi adaptado para diferentes idiomas e algumas pesquisas apontaram para sua utilidade em avaliar mudança em processos psicoterápicos individuais (Lepper \& Mergenthaler, 2005) e grupais (Fontao \& Mergenthaler, 2005). No Brasil, o TCM já foi utilizado juntamente com a Escala Rutgers de Progresso em Psicoterapia - RPPS (Rutgers Psychotherapy Progress Scale, 1992) para examinar a coincidência quanto aos momentos indicadores de progresso, segundo instrumentos de avaliação com diferentes origens: empírica e teórica (Yoshida \& Mergenthaler, 2010). Ele foi adaptado para a população de adolescentes em conflito com a lei, tendo em vista a peculiaridade da comunicação desses jovens (Khater \& Yoshida, 2011). Também se mostrou útil para verificar as relações entre tom emocional e mudança em processos terapêuticos (Yoshida \& Mergenthaler, 2011), identificar momentos que evidenciavam maior capacidade de mentalização pelo paciente (Ramires, Yoshida, Biazus, Khater \& Mergenthaler, 2012), bem como para verificar o tipo de intervenção e a orientação teórica do terapeuta e os momentos de mudança significativa em uma psicoterapia breve (Serralta, Yoshida, Khater, Domingues \& Mergenthaler, 2012).

$\mathrm{Na}$ presente pesquisa estabeleceu-se como objetivo avaliar possível associação entre a natureza das intervenções realizadas pela terapeuta e as respostas da paciente em momentos-chave de três fases do processo: inicial, medial e final. Tal objetivo demandava uma pesquisa de processo (Borsa \& Nunes, 2008), com o exame exaustivo das interações ocorridas entre a paciente e a terapeuta (Eells, 2007). Exigia também o envolvimento de juízes familiarizados com a classificação das intervenções do terapeuta (Fonagy \& Moran, 1993), além do emprego de material gravado em vídeo e áudio e transcrito para as devidas análises (Spence, 1993). Essas condições levaram os autores a optarem por um método de pesquisa baseado em caso único, procedimento extensamente empregado nas pesquisas de psicoterapia de orientação psicodinâmica e que pretende fornecer evidências relevantes para a prática clínica (Edwards, 2007; Messer, 2007).

O estudo, de caráter exploratório, partiu da hipótese de que o terapeuta "modularia" suas intervenções em função da fase da terapia (inicial, medial e final), considerando-se que cada uma delas é caracterizada por necessidades específicas do paciente. Esperava-se predomínio de intervenções suportivas na fase inicial do processo, predomínio de intervenções expressivas na fase medial e de suportivas na fase final do processo. Ademais, esperava-se predomínio de intervenções expressivas dentro dos ciclos terapêuticos, quando comparadas às intervenções fora dos ciclos terapêuticos, em cada sessão.

\section{Método}

\section{Participantes}

Paciente do sexo feminino, 23 anos de idade, estudante universitária com quadro clínico de transtornos alimentares. Terapeuta do sexo feminino, 24 anos de idade, um ano de formação e o mesmo tempo de experiência clínica. $O$ processo psicoterapêutico ocorreu sob supervisão em Psicoterapia Breve de Adultos, realizada por uma das autoras da pesquisa, cuja experiência clínica aproximava-se de 25 anos. $O$ atendimento foi realizado em clínica-escola da universidade em que a pesquisa foi realizada.

\section{Juizes}

Atuaram como juízes, três membros do grupo de pesquisa, familiarizados com a classificação das intervenções terapêuticas utilizada, uma doutora em psicologia, com 33 anos de experiência clínica, e dois alunos de doutorado, com cinco e quatro anos de experiência clínica. 


\section{Instrumentos}

Classificação das intervenções terapêuticas (IT) (Yoshida, Gatti, Enéas \& Coelho Filho, 1997) - compostas por 14 tipos, divididas segundo a natureza em: expressivas, suportivas e neutras. Para avaliar, cada intervenção é categorizada e a frequência relativa de cada tipo permite estimar o grau de apoio ou de expressividade predominante em um determinado momento ou fase da terapia. As intervenções expressivas objetivam facilitar a expressão e entendimento, pelo paciente, de seus problemas e conflitos. Intervenções que buscam manter o nível de funcionamento do paciente, mediante do apoio fornecido pelo psicoterapeuta, são ditas suportivas. E as intervenções de natureza neutra, localizadas no centro do continuum expressivo-suportivo, visam obter informações sobre o paciente, além de esclarecer aspectos relacionados ao processo psicoterápico. São consideradas intervenções expressivas: 1 . Interpretação - tornar consciente algo anteriormente inconsciente mediante a explicitação do desejo ou necessidade latente, a ansiedade e as defesas envolvidas na "ocultação" do desejo; 2. Confrontação - identificar algo que o paciente minimiza ou evita; 3. Clarificação - reformular ou reunir falas do paciente para torná-las mais coerentes; 4. Assinalamento - mostrar relações entre dados ou fatos não relacionados explicitamente pelo paciente. As intervenções suportivas são: 6. Validação empática - demonstrar a sintonia do terapeuta com aquilo que é falado ou expresso pelo paciente. Permite demonstrar que o terapeuta pode compreendê-lo naquele momento; 7. Recapitulação - resumir pontos essenciais da sessão ou do tratamento; 8. Conselhos e elogios - recomendar e reforçar certas atitudes; 11 . Fornecer informações - esclarecer aspectos técnicos desconhecidos do paciente. As intervenções neutras são: 5. Encorajamento a elaborar - pedir mais informações, de forma a manter o paciente focado no tema e leva-lo a aprofundar sua compreensão sobre ele; 9. Afirmação - comentários sucintos que apoiam comentários e atitudes do paciente; 10 . Interrogação - consultar e avaliar a consciência do paciente; 12. Intervenções interrompidas - início de intervenção do psicoterapeuta interrompida pelo paciente; 13 . Meta intervenção - objetiva esclarecer o motivo de ter feito alguma outra intervenção naquele momento da sessão ou tratamento; 14. Dar Enquadramento - intervenções relativas aos aspectos do enquadre terapêutico.

Modelo dos Ciclos Terapêuticos - TCM (Mergenthaler, 1996; 2008) - auxilia a identificar os momentos-chave durante uma sessão de psicoterapia, na medida em que avalia a expressão de emoções e de abstrações no discurso do paciente e do terapeuta. Por meio de gráficos gerados pelo software CM, é possível identificar a ocorrência de ciclos terapêuticos durante o atendimento. Esses são representados por uma sequência de padrões de emoção-abstração que inclui ao menos um bloco de palavras com o padrão conexão (muita emoção e muita abstração) e tem como limite inicial e final a ocorrência de um padrão de relaxamento (baixo tom emocional - igual ou inferior à média; baixa abstração). Quando não há um padrão de relaxamento anterior ao bloco em que ocorreu conexão, o início da sessão é considerado o início do ciclo (Lepper \& Mergenthaler, 2005).

\section{Resumo do processo terapêutico}

Queixa- falta de controle sobre a quantidade de alimentos ingeridos e sobre o impulso de forçar vômito após as refeições. Apesar de demonstrar intenção de eliminar esses sintomas, sentia-se muito desapontada por não obter sucesso nessas tentativas.

A partir das sessões iniciais, levantou-se como hipótese psicodinâmica que haveria associação entre os sintomas alimentares e o padrão de relacionamento estabelecido com as pessoas ao seu redor, nos quais sempre ocupava uma posição submissa às vontades do outro. Apenas no que se referia à alimentação parecia demonstrar "o controle" de manter dentro de si somente o que achava necessário. Diante desse entendimento, o objetivo do processo terapêutico foi de ajudar a paciente a desenvolver postura assertiva frente aos relacionamentos, elevando a autoestima e melhorado o sentimento de inferioridade, para que deixasse de "engolir" as imposições externas.

A psicoterapia, considerada bem sucedida, foi realizada em 11 sessões. Seus resultados foram avaliados clinicamente, quando se observou redução dos sintomas alimentares e melhoria no posicionamento da paciente diante das relações interpessoais.

Fatos importantes: nascida em uma família de classe média, passava por dificuldades financeiras após o adoecimento e desemprego do pai, o que a deixava muito preocupada. Não trazia lembranças da mãe durante as sessões, visto que esta havia falecido quando ainda era criança de pouca idade. Depois que a mãe faleceu, o pai enviou-a para ser cuidada pela avó, mas logo após, foi buscá-la, quando se casou novamente. A paciente apresentava muitas dificuldades em relacionar-se com sua madrasta e a filha dela. $\mathrm{Na}$ ocasião da 
terapia, estava namorando, sendo o namorado, a única pessoa percebida por ela como um ponto de apoio.

\section{Procedimento}

$\mathrm{O}$ processo psicoterapêutico obedeceu à demanda natural de pacientes que buscam psicoterapia na clínica-escola da universidade em que a pesquisa foi realizada. Uma vez convocada para o atendimento pela terapeuta, a paciente foi consultada sobre sua disponibilidade para participar da pesquisa. Informada sobre os objetivos e natureza da pesquisa, concordou com a colaboração voluntária. As sessões foram registradas em áudio e vídeo, mediante autorização formal prévia da paciente, por meio de assinatura a Termo de Consentimento Livre e Esclarecido. O projeto de pesquisa foi aprovado pelo Comitê de Ética em Pesquisa com seres Humanos na instituição em que se realizou a coleta de dados, sob o processo CEP/ UPM N 1165/09/2009 e CAAE No 0063.0.272.00009. Também foi obtida autorização dos responsáveis pela instituição para utilização do material. As sessões foram transcritas na íntegra, de acordo com as recomendações de Mergenthaler e Stinton (1992). A intenção, neste caso, foi manter a fidelidade ao texto, preservando ao máximo a entonação e inflexões do sujeito, e garantir acesso ao conjunto do material para as avaliações propostas.

Foram avaliadas seis sessões do processo de Psicoterapia breve psicodinâmica (PBP), dividido em fase inicial ( $1^{\mathrm{a}}$ e $2^{\mathrm{a}}$ sessões), medial ( $6^{\mathrm{a}}$ e $7^{\mathrm{a}}$ sessões), e final $\left(10^{a}\right.$ e $11^{a}$ sessões). Com base nas transcrições das sessões, os juízes avaliaram, de forma independente, cada uma das intervenções da psicoterapeuta, de acordo com a classificação das intervenções terapêuticas (IT). As transcrições também foram analisadas com o auxílio do $\mathrm{CM}$, dando origem aos gráficos do TCM.

Para análise dos resultados, foram utilizados os gráficos que fornecem os padrões de emoção-abstração provenientes da fala do paciente e do terapeuta. E quanto às intervenções, somente foram consideradas as expressivas e suportivas, visto que as neutras não costumam ensejar mudança. Para efeito de rejeição das hipóteses estipulou-se previamente nível de significância $(\alpha) \mathrm{p} \leq 0,05$.

\section{Resultados}

De acordo com o TCM, foram identificados 14 ciclos terapêuticos nas seis sessões analisadas, sendo que na sessão 2 ocorreram quatro ciclos e nas sessões 1 , 6, 7, 10 e 11, dois ciclos em cada. A distribuição de frequência das intervenções nas sessões, como um todo, fora e dentro dos ciclos terapêuticos, é apresentada na Tabela 1. As frequências de intervenções dentro dos ciclos de cada sessão foram somadas e apenas os totais foram considerados.

A seguir, foram estimadas as significâncias das diferenças entre as intervenções expressivas e suportivas de cada sessão como um todo e a significância das diferenças entre a ocorrência de intervenções expressivas e suportivas dentro dos ciclos de cada sessão. Para tanto, empregou-se a prova de qui-quadrado de aderência $\left(\chi^{2}\right)$ de uma amostra, hipótese bicaudal (Siegel, 1981) (Tabela 2).

Não foram observadas diferenças estatisticamente significantes entre o número de intervenções expressivas e suportivas nas duas sessões iniciais, na $6^{a}$ e $10^{a}$ sessões. $\mathrm{Na} 7^{a}$ sessão predominaram as

Tabela 1. Distribuição das intervenções terapêuticas durante as sessões e dentro e fora dos ciclos terapêuticos

\begin{tabular}{|c|c|c|c|c|c|c|c|c|c|c|c|c|}
\hline \multirow[b]{3}{*}{ Sessão 1} & \multicolumn{4}{|c|}{$\begin{array}{l}\text { Total de intervenções } \\
\text { durante a Sessão }\end{array}$} & \multicolumn{4}{|c|}{$\begin{array}{l}\text { Intervenções dentro dos } \\
\text { ciclos terapêuticos }\end{array}$} & \multicolumn{4}{|c|}{$\begin{array}{l}\text { Intervenções fora dos } \\
\text { ciclos terapêuticos }\end{array}$} \\
\hline & \multicolumn{2}{|c|}{ Expressivas } & \multicolumn{2}{|c|}{ Suportivas } & \multicolumn{2}{|c|}{ Expressivas } & \multicolumn{2}{|c|}{ Suportivas } & \multicolumn{2}{|c|}{ Expressivas } & \multicolumn{2}{|c|}{ Suportivas } \\
\hline & 13 & $34 \%$ & 25 & $66 \%$ & 4 & $36 \%$ & 7 & $64 \%$ & 9 & $33 \%$ & 18 & $67 \%$ \\
\hline Sessão 2 & 34 & $49 \%$ & 36 & $51 \%$ & 4 & $25 \%$ & 12 & $75 \%$ & 30 & $56 \%$ & 24 & $44 \%$ \\
\hline Sessão 6 & 36 & $42 \%$ & 50 & $58 \%$ & 24 & $60 \%$ & 16 & $40 \%$ & 12 & $26 \%$ & 34 & $74 \%$ \\
\hline Sessão 7 & 49 & $61 \%$ & 31 & $39 \%$ & 26 & $57 \%$ & 20 & $43 \%$ & 23 & $68 \%$ & 11 & $32 \%$ \\
\hline Sessão 10 & 55 & $59 \%$ & 38 & $41 \%$ & 4 & $80 \%$ & 1 & $20 \%$ & 51 & $58 \%$ & 37 & $42 \%$ \\
\hline Sessão 11 & 44 & $34 \%$ & 85 & $66 \%$ & 13 & $37 \%$ & 22 & $63 \%$ & 31 & $33 \%$ & 63 & $67 \%$ \\
\hline
\end{tabular}


Tabela 2. Valores dos qui-quadrados de pertinência $\left(\chi_{0}^{2}\right)$ relativos à ocorrência das intervenções terapêuticas

\begin{tabular}{lcccccc}
\hline Trecho da sessão & $1^{\text {a }}$ sessão & $2^{\text {a }}$ sessão & $6^{\text {a }}$ sessão & $7^{\text {a }}$ sessão & $10^{\text {a }}$ sessão & $11^{\text {a }}$ sessão \\
\hline Total & 3,789 & 0,006 & 2,279 & $\mathbf{4 , 0 5 * *}$ & 3,107 & $\mathbf{1 3 , 0 3 1 * * *}$ \\
Dentro dos ciclos & 0,818 & $\mathbf{4 , 0 * * *}$ & 1,6 & 0,782 & 1,8 & 2,314 \\
\hline
\end{tabular}

$* \chi_{c}^{2}=3,841 ; * *$ predomínio expressivas; $* * *$ predomínio suportivas

expressivas $\left[\chi^{2}(1 \mathrm{gl}, \mathrm{bi})=4,050\right]$ e na $11^{\mathrm{a}}$, as suportivas $\left[\chi^{2}(1 \mathrm{gl}, \mathrm{bi})=13,031\right]$ (Tabela 2$)$. Em relação às diferenças entre as duas modalidades de intervenção dentro dos ciclos terapêuticos, apenas na $2^{\mathrm{a}}$ sessão predominaram as suportivas $\left[\chi^{2}(1 \mathrm{gl}, \mathrm{bi})=4,0\right]$, enquanto nas demais as diferenças não foram estatisticamente significantes (Tabela 2).

Em relação aos temas tratados nos trechos das sessões relativos aos ciclos terapêuticos, na $1^{\mathrm{a}}$ e $2^{\mathrm{a}}$ sessões estão ligados à identificação de aspectos da vida da paciente, em especial seus relacionamentos interpessoais. Desse modo, foi possível identificar a necessidade da paciente em agradar os outros e em ser reconhecida pelo que faz. Quanto aos ciclos referentes às sessões mediais, $6^{\mathrm{a}}$ e $7^{\mathrm{a}}$, estes correspondem às temáticas delimitadas pelo foco terapêutico, ou seja, busca-se uma expansão da consciência da paciente na relação entre os sintomas bulímicos e dificuldades relacionais. Já nos ciclos da $10^{\mathrm{a}}$ e $11^{\mathrm{a}}$, observa-se a realização de uma retrospectiva do processo terapêutico, bem como o trabalho de término, no qual a terapeuta busca estabelecer ligações entre as dificuldades de separação vividas pela paciente no decorrer do seu desenvolvimento e o momento específico do processo terapêutico.

\section{Discussão}

O trabalho teve como objetivo estudar empiricamente possível relação entre a modalidade de intervenção do terapeuta - expressiva ou suportiva - e a fase do processo terapêutico. Também se explorou possível relação entre a modalidade de intervenção e a ocorrência de ciclos terapêuticos, considerados momentos clinicamente relevantes e associados a mudanças (Mergenthaler, 1996; 2008). A expectativa era a de que o terapeuta "modularia" suas intervenções em função das necessidades do paciente em cada uma das etapas em que usualmente se dividem as psicoterapias: fase inicial, medial e final (Enéas \& Yoshida, 2012). Nesse sentido, esperava-se predomínio de intervenções suportivas nas fases inicial e final e predomínio de intervenções expressivas na fase medial. E, especificamente em relação às intervenções dentro dos ciclos terapêuticos, esperava-se predomínio de intervenções expressivas.

$\mathrm{Na}$ fase inicial, embora tenham predominado as intervenções suportivas nas duas sessões como um todo (respectivamente, 66\% e 51\%) (Tabela 1), a diferença em relação às expressivas não foi estatisticamente significante $\left[\chi^{2}(1 \mathrm{gl}, \mathrm{bi})=3,789\right]$ (Tabela 2). $\mathrm{E}$ em relação às intervenções dentro dos ciclos, também houve equilíbrio de expressivas e suportivas na $1^{\text {a }}$ sessão $\left[\chi^{2}(1 \mathrm{gl}, \mathrm{bi})=0,818\right]$ (Tabela 2$)$ e predomínio de suportivas na $2^{\mathrm{a}}$ sessão[ $\left.\chi^{2}(1 \mathrm{gl}, \mathrm{bi})=4,0\right]$. Ou seja, na fase inicial, a terapeuta recorreu a intervenções de natureza expressiva em proporção semelhante às suportivas, contrariando a expectativa teórica.

As intervenções expressivas que foram utilizadas na fase inicial diziam respeito a uma primeira tentativa de compreensão psicológica sobre o transtorno alimentar da paciente. Observa-se a tentativa da terapeuta em resumir os pontos essenciais levantados no decorrer da avaliação, buscando fazer uma ligação entre a queixa apresentada pela paciente, vista a princípio apenas como de ordem física, às suas dificuldades de cunho psicológico e emocional, mais especificamente seu padrão relacional mal-adaptativo (Luborsky \& Crits-Christoph, 1998), o que se constituiu o foco do processo terapêutico.

As intervenções suportivas, nessa primeira etapa do processo, tiveram função de demonstrar o interesse da terapeuta em compreender as dificuldades e motivos que levaram a paciente a buscar auxílio psicoterápico. Além disso, elas podem ser vistas como uma estratégia em estabelecer boa aliança entre elas. Dessa forma, a compreensão psicodinâmica expressa no foco sugerido pelo terapeuta, a partir da $2^{\mathrm{a}}$ sessão, teria maior chance de ser aceita pela paciente, especialmente pelo fato de esta se sentir compreendida em seu sofrimento. Isso vai ao encontro das expectativas teóricas de que um bom vínculo precocemente estabelecido entre paciente e psicoterapeuta pode gerar uma relação de confiança e 
compromisso mútuo, que contribui positivamente para desenvolvimento e sucesso do tratamento (Botella e colaboradores, 2008; Fernandez e colaboradores, 2008).

$\mathrm{Na}$ fase medial, manteve-se o equilíbrio entre as intervenções suportivas e expressivas na $\sigma^{\text {a }}$ sessão $\left[\chi^{2}(1 \mathrm{gl}, \mathrm{bi})=2,279\right]$ (Tabela 2$)$ e inclusive dentro dos ciclos $\left[\chi^{2}(1 \mathrm{gl}, \mathrm{bi})=1,6\right]$ (Tabela 2$)$. Na $7^{\mathrm{a}}$ sessão, conforme o esperado, prevaleceram as intervenções expressivas $\left[\chi^{2}(1 \mathrm{gl}, \mathrm{bi})=4,05\right]$ (Tabela 2$)$, mas não dentro dos ciclos, quando a diferença não foi significante $\left[\chi^{2}(1 \mathrm{gl}, \mathrm{bi})=0,782\right]$ (Tabela 2).

O trabalho da terapeuta foi no sentido de levar a paciente a compreender que, na tentativa de agradar os outros, acabava ficando quieta, para não gerar desavenças. A terapeuta, por meio de intervenções de natureza expressivas - predominantes dentro da $7^{\mathrm{a}}$ sessão $\left[\chi^{2}(1 \mathrm{gl}, \mathrm{bi})=4,05\right]$ (Tabela 2$)$, tenta ajudá-la a compreender melhor esse funcionamento, o que é aceito pela paciente, que passa a desenvolver mais o tema tratado. Observa-se que, de acordo com a perspectiva teórica, a fase medial foi marcada pelos esforços da psicoterapeuta em direcionar o trabalho para as questões relacionadas ao foco (Enéas \& Yoshida, 2012). Nesse sentido, o uso das intervenções expressivas objetivava ampliar a consciência da paciente, mediante a identificação de seus conflitos inconscientes e da demonstração das relações entre suas dificuldades atuais, expressas em sua queixa, e seus conflitos de ordens psicológicas, que se estabeleceram no decorrer de seu desenvolvimento, especialmente na infância.

$O$ equilíbrio entre intervenções expressivas e suportivas também foi observado na $10^{\mathrm{a}}$ sessão e nos seus ciclos, assim como nos ciclos da $11^{a}$ sessão. Apenas na $11^{a}$ sessão houve predomínio de intervenções suportivas, conforme o esperado. Os temas dos ciclos das sessões finais $\left(10^{\mathrm{a}}\right.$ e $\left.11^{\mathrm{a}}\right)$ dizem respeito ao término do processo, quando há oportunidade para realização de recapitulações, em que se revê o conteúdo trabalhado, e a psicoterapeuta se esforça para que a paciente guarde com ela os ganhos obtidos e não ocorra a reativação de defesas e recaídas, com vistas a perpetuar a relação terapêutica (Enéas \& Rocha, 2011).

Nesse trabalho de término, houve especial atenção ao processo de separação, pois este reatualizava as questões referentes à perda da mãe da paciente, bem como a perda do pai na infância, quando foi enviada para morar com a avó. A explicitação desses conteúdos exigiu da psicoterapeuta não só intervenções de natureza expressiva, mas também uma sólida base suportiva, para que a paciente aceitasse tais formulações e associasse os conflitos infantis às dificuldades transferenciais, resultando em certo equilíbrio na utilização das intervenções de natureza expressiva e suportiva dentro dos ciclos $\left(\left[\chi^{2}(1 \mathrm{gl}, \mathrm{bi})=1,8\right]\right.$ na $10^{\mathrm{a}}$ sessão e na $11^{a}$ sessão $\left[\chi^{2}(1 \mathrm{gl}, \mathrm{bi})=2,314\right]$ (Tabela 2$)$. Já a utilização de estratégia interventiva predominantemente suportiva, na última sessão do processo $\left[\chi^{2}(1 \mathrm{gl}, \mathrm{bi})=13,031\right]$ (Tabela 2), ocorreu num momento em que a terapeuta tenta ajudar a paciente a internalizar tais ganhos. Nesse momento, efetivamente, já não se tinha como objetivo o levantamento de novos temas, assim como não se buscava grandes elaborações, o que corrobora dados da literatura que apontam que em cada momento do processo são exigidos do terapeuta diferentes manejos, expressos na natureza da intervenção utilizada (Krause e colaboradores, 2007).

Em termos gerais, os resultados não corroboraram as expectativas teóricas. A suposição de que haveria relação entre a fase do processo e o predomínio de uma modalidade específica de intervenções - suportiva no início e no final e expressiva na fase medial - não se sustentou. Desde o início, a terapeuta recorreu às duas modalidades de intervenção, guiada não apenas pela necessidade de acolher o sofrimento psíquico da paciente e garantir uma boa aliança terapêutica, mas também para explicitar a hipótese psicodinâmica e demarcar o foco do atendimento. O recurso às intervenções tanto suportivas quanto expressivas manteve-se na fase medial e final, sugerindo que as intervenções da terapeuta se deram muito mais em função de objetivos específicos de cada momento do processo, do que de objetivos amplos, aplicáveis a toda a fase, seja ela inicial, medial ou final.

Este estudo possibilitou ainda uma reflexão mais ampla sobre a formação dos ciclos terapêuticos, uma vez que sua ocorrência durante o processo não parece estar ligada diretamente ao tipo de intervenção do terapeuta (pois ambas as intervenções compõem os ciclos). Aparentemente, a ocorrência de momentos clinicamente significantes seria decorrência de todo um trabalho realizado previamente, e que enseja a possibilidade de mudança (Mergenthaler, 1996; 2008). E nesses momentos, como nos demais, o terapeuta parece se pautar muito mais por sua compreensão do que é vivido e verbalizado pelo paciente a cada momento.

\section{Conclusões e considerações finais}

Ainda que o método de estudo de caso único atendesse aos objetivos da pesquisa, algumas limitações 
dos instrumentos de avaliação podem ter contribuído para que os resultados não corroborassem plenamente as expectativas teóricas. Os resultados suscitaram algumas críticas, que devem ser ponderadas em pesquisas futuras.

O emprego do TCM nas diferentes fases do processo terapêutico pôs à prova o caráter mecânico do modelo, que segue a mesma lógica para identificar um ciclo terapêutico, independentemente do momento vivido. Ademais, um ciclo na fase inicial teria o mesmo "peso relativo" de outro na fase medial ou final, quando todo o contexto, - que inclui a compreensão do paciente em relação às suas dificuldades; a qualidade do vínculo com o terapeuta e as expectativas de mudança -, já teria sido profundamente modificado. Essas características, que podem ter funcionado como fatores de viés na presente pesquisa, não haviam sido reconhecidas, até o momento, pelos autores que empregaram o modelo em seus estudos (por exemplo, Fontao \& Mergenthaler, 2005; Khater \& Yoshida, 2011; Lepper \& Mergenthaler, 2005; Mergenthaler, 1996; 2008; Yoshida \& Mergenthaler, 2010; 2011).

Quanto ao critério de classificação das intervenções - divididas em suportivas e expressivas -, pode ter sido também um fator indutor de viés, na medida em que não se considerou o peso das intervenções específicas, utilizadas pela terapeuta em cada fase. Cada uma das duas categorias abrange um rol maior de possibilidades de intervenção, que têm funções e impactos diversos sobre a paciente.

Apesar de os resultados não terem corroborado plenamente as hipótese levantadas, acredita-se que as estratégias interventivas do terapeuta constituam um fator de máxima importância em qualquer processo terapêutico. Elas ajudam o paciente a perceber e refletir sobre seus conflitos e dificuldades, ora fornecendo-lhe suporte e compreensão de sua condição, ora instigando-o a estabelecer associações entre sua experiência afetiva e cognitiva. Na prática clínica, elas não ocorrem ao acaso, mas são moduladas pelas respostas do paciente, bem como pelas tarefas específicas do terapeuta, tais como estabelecer o foco, trabalhar o término, entre outras. Ou seja, as intervenções são feitas dentro de um contexto relacional, em que as respostas do terapeuta controlam e são controladas pelas respostas do paciente, nas diferentes etapas do processo psicoterápico (Krause e colaboradores, 2007). A complexidade do processo psicoterapêutico, que é intuída pelo clínico e que vem sendo apontada por inúmeras pesquisas da área (Angus e colaboradores, 2010; Botella e colaboradores, 2008), continua a ser um desafio a todos que buscam evidências empíricas para compreender os fatores nele envolvidos.

\section{Referências}

Angus, L., Hayes, J. A., Anderson, T., Ladany, N., Castonguay, L. G., \& Muran, J. C. (2010). Future directions: emerging opportunities and challenges in psychotherapy research. Em L. G. Castonguay, J. C. Muran, L. Angus, J. A. Hayes, N. Ladany, \& T. Anderson (Eds.), Bringing psychotherapy research to life: understanding change through the work of leading clinical researchers (pp. 353-362). Washington, DC: American Psychological Association Press.

Borsa, J. C. \& Nunes, M. L. T. (2008). O sujeito/pesquisador na pesquisa em psicologia clínica. Psicologia Argumento, 26(52), 47-54.

Botella, L., Corbella, S., Belles, L., Pacheco, M., Gomez, A. M., Herrero, O., Ribas, E. \& Pedro, N. (2008). Predictors of therapeutic outcome and process. Psychotherapy Research, 18(5), 535-542.

Castonguay, L. G., \& Beutler, L. E. (2005). Principles of therapeutic change that work. New York: Oxford University Press.

Edwards, D. J. A. (2007). Collaborative versus advesarial stances in scientific discourse: implications for the role of systematic case studies in the development of evidence-based practice in psychotherapy. Pragmatic Case Studies in Psychotherapy [Online], 3(1), 6-34. Disponível: http://pcsp.libraries.rutgers.edu.

Eells, T. D. (2007). Generating and generalizing knowledge about psychotherapy from pragmatic case studies. Pragmatic Case Studies in Psychotherapy [Online], 3(1), 35- 54. Disponível: http:/ / pcsp.libraries. rutgers.edu.

Enéas, M. L. E. \& Rocha, G. M. A. (2011). Momentos decisivos em psicoterapia breve: manejo do término. Em S. F. S. Cavalini \& C. Batista (Orgs.), Clínica psicodinâmica: olhares contemporâneos (pp. 129-144). São Paulo: Vetor.

Enéas, M. L. E. \& Yoshida, E. M. P. (2012). Psicoterapia Breve Psicodinâmica de Adultos. Em M. L. E. Lipp \& E. M. P. Yoshida (Orgs.), Psicoterapias breves nos diferentes estágios evolutivos (pp. 156-176). São Paulo: Casa do Psicólogo. 
Fernandez, P. M. S., Mella, M. F. R., Chenevard, C. L., García, A. E. E., Cáceres, D. E. I., \& Vergara, P. A. M. (2008). Variables inespecíficas em psicoterapia. Terapia Psicológica, 26(1), 89-98.

Fiorini, H. J. (1995). Teoria e técnicas psicoterápicas. Rio de Janeiro: Francisco Alves.

Fonagy, P. \& Moran, G. (1993). Selecting single case research designs for clinicians. Em N. E. Miller, L. Luborsky, J. P. Barber, \& J. P. Docherty (Orgs.), Psychodynamic treatment research: a handbook for clinical practice (pp. 62-95). Nova Iorque: Basic Books.

Fontao, M., \& Mergenthaler, E. (2005). El concepto de "insight" en la psicoterapia de grupo: aporte a su clariflcación mediante el uso de métodos de análisis de texto computarizado y un modelo de la actividad verbal en los procesos terapêuticos. Revista del Instituto de Investigaciones de la Facultad de Psicología, 10(3), 55-71.

Gabbard, G. O. (1998). Psiquiatria psicodinâmica na prática clínica (A. E. Fillman, trad.). Porto Alegre, Artes Médicas. (Original publicado em 1994).

Khater, E., \& Yoshida, E. M. P. (2011). Desenvolvimento de um dicionário personalizado do modelo dos ciclos terapêuticos (TCM) para adolescentes em conflito com a lei. Psicologia: Teoria e Prática, 13(2), 168-181.

Krause, M., de la Parra, G., Arístegui, R., Dagnino, P., Tomicic, A., Valdés, N., Echávarri, O., Strasser, K., Reyes, L., Altimir, C., Ramirez, I. Vilches, O., \& Ben-Dov, P. (2007). The evolution of therapeutic change studied through generic change indicators. Psychotherapy Research, 17(6), 673-689.

Lepper, G., \& Mergenthaler, E. (2005). Exploring group process. Psychotherapy Research, 15(4), 433-444.

Luborsky, L. (1984). Principles of Psychoanalytic psychotherapy: a manual for supportive-expressive treatment. Nova Iorque: Basic Books.

Luborsky, L. \& Crits-Christoph, P. (1998). Understanding transference: the CCRT method. ( $\left.2^{\mathrm{a}} \mathrm{ed}\right)$. Nova Iorque: Basic Books.

Mergenthaler, E. (1996). Emotion- abstraction patterns in verbatim protocols: a new way of describing psychotherapeutic processes. Journal of Consulting and Clinical Psychology, 64(60), 1306-1315.
Mergenthaler, E. (2008). Resonating minds: a school-independent theoretical conception and its empirical application to psychotherapeutic processes. Psychotherapy Research, 18(2), 109-126.

Mergenthaler, E., \& Stinton, C.H. (1992). Psychotherapy transcription standards. Psychotherapy Research, 2, 125-142.

Messer, S. B. (2007). Psychoanalytic Case studies and the pragmatic case study method. Pragmatic Case Studies in Psychotherapy [Online], 3(1), 55-58. http:/ / pcsp.libraries.rutgers.edu.

Messer, S. B. \& Warren, C. S. (1995). Models of brief psychodynamic therapy: a comparative approach. Nova Iorque: Guilford Press.

Ramires, V. R. R., Yoshida, E. M. P., Biazus, C., Khater, E., \& Mergenthaler, E. (2012). Clinically significant moments and mentalization based interventions in a psychodynamic psychotherapy with a female adolescent with symptoms of depression. Em 41st Annual Meeting of SPR. Book of Abstracts 41st Annual Meeting of SPR (pp. 122). Asilomar, Califórnia, Estados Unidos

Rutgers Psychotherapy Progress Scale - RPPS (1992). Scoring manual. Manuscrito não publicado. Rutgers University, Nova Jersey.

Serralta, F. B., Yoshida, E. M. P., Khater, E., Domingues, A., \& Mergenthaler, E. (2012). Adherence to the "psychodynamic prototype" in key moments of a brief psychotherapy. Em 43rd Annual Meeting os SPR. Book of Abstracts 43rd Annual Meeting of SPR (pp. 110-111). Virginia Beach, Virginia, Estados Unidos.

Siegel, S. (1981). Estatística não-paramétrica para as ciências do comportamento. São Paulo: MGHB.

Spence, D. P. (1993). Traditional case studies and prescriptions for improving them. Em N. E. Miller, L. Luborsky, J. P. Barber, \& J. P. Docherty (Orgs.), Psychodynamic treatment research: a handbook for clinical practice (pp. 37-52). Nova Iorque: Basic Books.

Yoshida, E. M. P., Gatti, A. L., Enéas, M. L. E., \& Coelho Filho, J. G. (1997). Aliança terapêutica, transferência e motivação num processo de psicoterapia breve. Mudanças: Vértices de Observação, 7, 141-154.

Yoshida, E. M. P, \& Mergenthaler, E. (2010). Empirical and theoretical inspired instruments to measure 
progress in psychotherapy: Do they match? Em 41 st Annual Meeting of SPR. Book of Abstracts 41st Annual Meeting of SPR (pp. 29), Asilomar, California, Estados Unidos.

Yoshida, E. M. P., \& Mergenthaler, E. (2011). Coincidência de conexão do tom emocional com abstração e o resultado psicoterapêutico. Estudos de Psicologia (Campinas), 28, 35-43.

Recebido em: 03/03/2013

Reformulado em: 28/10/2013

Segunda reformulação em: 03/01/2014

Aprovado em: 07/02/2014

Sobre os autores:

Eduardo Khater é mestre em Psicologia como Profissão e Ciência pela Pontifícia Universidade Católica de Campinas, doutorando em Psicologia na mesma Universidade. Membro do grupo de pesquisa Psicoterapia Breve Psicodinâmica: avaliação de mudança e instrumentos de medida e membro da Society for Psychotherapy Research. Tem experiência na área de Psicologia Clínica e Social, Terceira idade, Adolescentes em conflito com a lei, Docência em ensino superior e Educação Infantil.

Evandro Morais Peixoto é graduado em Psicologia pela Universidade Presbiteriana Mackenzie. Mestre em Psicologia como Profissão e Ciência pela Pontifícia Universidade Católica de Campinas, doutorando em Psicologia na mesma universidade, membro do grupo de pesquisa Psicoterapia Breve Psicodinâmica: avaliação de mudança e instrumentos de medida e membro da Society for Psychotherapy Research (SPR). Possui experiência em pesquisas de processos em psicoterapias e Psicologia do Esporte.

Giovanna Corte Honda é mestre em Psicologia como Profissão e Ciência pela Pontifícia Universidade Católica de Campinas, doutoranda em Psicologia na mesma universidade, membro do grupo de pesquisa Psicoterapia Breve Psicodinâmica: avaliação de mudança e instrumentos de medida e membro da Society for Psychotherapy Research (SPR). Possui experiência em pesquisas de processos em psicoterapias.

Maria Leonor Espinosa Enéas é graduada em Psicologia pela Faculdade de Filosofia Ciências e Letras de Ribeirão Preto da Universidade de São Paulo, mestre em Psicologia pela Pontifícia Universidade Católica de Campinas e doutora em Psicologia pela mesma universidade. Membro da Society for Psychotherapy Research (SPR), é professora adjunta II da Universidade Presbiteriana Mackenzie e membro do conselho Editorial da Revista Psicologia: Teoria e Prática.

Elisa Medici Pizão Yoshida é mestre e doutora em Psicologia pela Universidade de São Paulo, pós doutora pela Université de Montréal, Canadá, professora no Programa de Pós Graduação Stricto Sensu em Psicologia da PUC Campinas. Lider do Grupo de Pesquisa do Diretório do CNPQ: Psicoterapia breve psicodinâmica: avaliação de mudança e instrumentos de medida, também é presidente do Capítulo Latino-Americano da Society for Psychotherapy Research - SPR (2007 - 2008).

\section{Contato com os autores:}

Pontifícia Universidade Católica de Campinas

Centro de Ciências da Vida - Curso de Pós-Graduação em Psicologia

Av. John Boyd Dunlop, s/n - Jardim Ipaussurama

CEP 13060-904 - Campinas, SP - Brasil 